\title{
The Development of a Preprocessing Circuit Using Clocked CMOS Neuron Inverters for a Retinal Prosthesis System
}

\author{
Kei Eguchi ${ }^{\mathrm{a},}{ }^{,}$, Ponglert Rattanachinalai ${ }^{\mathrm{a}}$, Kanji Abe $^{\mathrm{a}}$, Yujiro Harada ${ }^{\mathrm{b}}$, Kuniaki Fujimoto ${ }^{\mathrm{b}}$ \\ aDepartment of Information Electronics, \\ Fukuoka Institute of Technology, \\ 3-30-1, Wajiro-Higashi, Higashi-ku, Fukuoka 811-0295, Japan \\ bDepartment of Electronics and Intelligent Systems Engineering, \\ Tokai University \\ 9-1-1, Toroku, Higashi-ku, Kumamoto 862-8652, Japan \\ *Corresponding Author: eguti@fit.ac.jp
}

\begin{abstract}
In implantable electronic medical devices, low power consumption and small size are important requirements to design efficient implantable circuits. As a preprocessing circuit of the retinal prosthesis system, this paper presents two types of image processing circuits: a CMOS median filter and a CMOS binarizing circuit. Owing to the CMOS circuit design utilizing clocked neuron CMOS inverters, the proposed circuits can achieve median filtering and binarizing of the captured image without arithmetic logic circuits. Furthermore, the through-current of neuron CMOS inverters can be suppressed by adjusting the duty cycle of clock pulses. Therefore, the proposed circuits achieve not only simple circuit configuration but also low power consumption. The results of the simulation program with integrated circuit emphasis (SPICE) simulation demonstrate the effectiveness of the proposed circuits.
\end{abstract}

Keywords: Median filter circuits, Image binarization, Image resizing, Neuron MOSFETs, Image processing, Implantable electronics.

\section{Introduction}

In the field of biomedical engineering, the demand of implantable electronic medical devices is growing rapidly. Among others, the development of a retinal prosthesis system ${ }^{(1-3)}$ is one of the most challenging issues to support partial or total blind patients. In the retinal prosthesis system, an image data captured by an external camera is transmitted to an IC chip implanted near retina by wireless ${ }^{(2,3)}$. Hence, the retinal prosthesis system consists of a camera, signalprocessing units, a wireless transmitter \& receiver, and an implanted stimulator IC chip. However, the existing retinal prosthesis system is bulky and not power-efficient. To develop an efficient retinal prosthesis system with small size and low power consumption, firstly, we focused on the preprocessing circuit to perform median filtering, resizing, and binarizing of the input image.

In previous studies, many types of the preprocessing circuits have been proposed. For example, Kalali et al. implemented an adaptive median filter into a Field Programmable Gate Array (FPGA) chip ${ }^{(4)}$. Due to the low complexity 2D adaptive median filter algorithm, low power consumption can be realized by Kalali's circuit. In this way, the efficient preprocessing circuit can be realized easily by utilizing embedded systems such as FPGA, Intel Edison, Aduino, and so on. However, the hardware approach based on the embedded system is too bulky for the retinal prosthesis system. Different from these approaches based on embedded systems, Lee et al. proposed a bit-level scalable median filter ${ }^{(5)}$. The bit-level scalable median filter is suitable for IC implementation, because it can be designed by CMOS technology. Following this, Yamasaki et al. suggested a high speed median filter using floating-gateMOS-based low-power majority voting circuits ${ }^{(6)}$. Due to the floating gate MOS technology, the median filter proposed by Yamasaki et al. can achieve not only low power consumption but also high speed operation. The floating gate MOSFET is referred to as a neuron MOSFET ${ }^{(7-10)}$. It is 
known that the floating gate MOS technology can achieve high-speed operation, small size, and low power consumption $(6-10)$. However, in order to realize more efficient implantable circuit, there is still room for improvement in the point of power consumption.

In this paper, we propose a novel preprocessing circuits using clocked CMOS neuron inverters: CMOS median filter and CMOS binarizing circuit. Owing to the CMOS circuit design utilizing clocked neuron CMOS inverters, the proposed circuits can achieve median filtering and binarizing of the captured image without arithmetic logic circuits, such as adder, subtractor, multiplier, and divider. Furthermore, the through-current of neuron CMOS inverters can be suppressed by adjusting the duty cycle of clock pulses. Therefore, the proposed circuits achieve not only simple circuit configuration but also low power consumption. To clarify the characteristics of the proposed circuits, SPICE (simulation program with integrated circuit emphasis) simulations are performed.

The rest of this paper is organized as follows. In section 2, the proposed CMOS median filter is described. Next, in section 3, the proposed binarizing circuit is presented. Simulation results are shown in section 5. Finally, conclusion and future work are drawn in section 6 .

\section{CMOS Median Filter}

\subsection{Operation Principle}

The operation principle of the proposed median filter is based on the binary search algorithm. To obtain the $k$-th $(k=0$, $\ldots, N-1)$ bit of the median value $M_{i}[k](i=1, \ldots, m \times n)$ from the input image of $m \times n$ pixels, the following operation is performed:

$$
M_{i}[k]=\left\{\begin{array}{lll}
1, & \text { if } & \sum_{j \in W} \frac{P_{j}[k]}{W}>0.5 \\
0, & \text { if } & \sum_{j \in W} \frac{P_{j}[k]}{W} \leq 0.5
\end{array},\right.
$$

where $P_{j}[k]$ is a value of the $j$-th pixel and $W$ is the window size. According to the median value $M_{i}[k]$, the value of the $i$ th pixel $P_{i}[k]$ is changed as follows:

$$
\begin{aligned}
& P_{i}[k-1]=\cdots=P_{i}[0]
\end{aligned}
$$

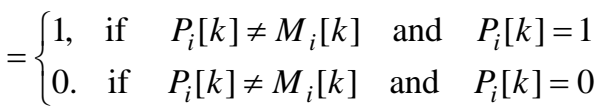

By iterating the operation of (1) and (2) from $k=N-1$ to 0 , the median value of $P_{i}$ is derived.

To help readers' understanding, an example of the median detection is demonstrated in Table 1 , where the window size $W=5$ and the bit-length $N=4$. First, in the case of $k=3$, the majority group is $P_{1}[3], P_{3}[3]$, and $P_{4}[3]$. Therefore, we have $M_{i}[3]=1$, because the majority is " 1 ". On the other hand, the remaining bits of $P_{2}$, and $P_{5}$ are all changed to " 0 ". Next, in the case of $k=2$, the majority group is $P_{1}$ [2], $P_{2}$ [2], $P_{3}$ [2] , and $P_{5}$ [2]. Therefore, we have $M_{i}[2]=0$, because the majority is " 0 ". On the other hand, the remaining bits of $P_{4}$ are all changed to " 1 ". In the same way, the median value $M_{i}[1]$ and $M_{i}[0]$ are obtained by iterating these processes.

\begin{tabular}{|c|c|c|c|c|}
\hline$(W=5)$ & $\begin{array}{c}k=3 \\
(\mathrm{MSB})\end{array}$ & $k=2$ & $k=1$ & $\begin{array}{c}k=0 \\
(\mathrm{LSB})\end{array}$ \\
\hline$P_{1}=\{1,0,0,1\}$ & $\begin{array}{l}\{1,0,0,1\} \\
\text { (Major) }\end{array}$ & $\begin{array}{c}\{1,0,0,0,1\} \\
\text { (Major) }\end{array}$ & $\begin{array}{c}\{1,0,0,1\} \\
\text { (Major) }\end{array}$ & $\begin{array}{r}\{1,0,0,1\}\} \\
\text { (Major) }\end{array}$ \\
\hline$P_{2}=\{0,1,1,1\}$ & $\begin{array}{l}\{[0,0,0,0\} \\
\text { (Minor) }\end{array}$ & $\begin{array}{c}\{0,0,0,0,0\} \\
\text { (Major) }\end{array}$ & $\begin{array}{c}\{0,0,0,0\} \\
\text { (Major) }\end{array}$ & $\begin{array}{r}\{0,0,0,0\} \\
\text { (Minor) }\end{array}$ \\
\hline$P_{3}=\{1,0,1,0\}$ & $\begin{array}{l}\{1,0,1,0\} \\
\text { (Major) }\end{array}$ & $\begin{array}{c}\{1,0,1,0\} \\
\text { (Major) }\end{array}$ & $\begin{array}{c}\{1,0,1,1\} \\
\text { (Minor) }\end{array}$ & $\begin{array}{r}\{1,0,1,1\}\} \\
\text { (Major) }\end{array}$ \\
\hline$P_{4}=\{1,1,0,0\}$ & $\begin{array}{l}\{1,1,0,0\} \\
\text { (Major) }\end{array}$ & $\begin{array}{l}\{1,1,1,1\} \\
\text { (Minor) }\end{array}$ & $\begin{array}{c}\{1,1,1,1\} \\
\text { (Minor) }\end{array}$ & $\begin{array}{r}\{1,1,1,1\}\} \\
\text { (Major) }\end{array}$ \\
\hline$P_{5}=\{0,1,0,0\}$ & $\begin{array}{l}\{0,0,0,0\} \\
\text { (Minor) }\end{array}$ & $\begin{array}{l}\{0,0,0,0,0\} \\
\text { (Major) }\end{array}$ & $\begin{array}{l}\{0,0,0,0\} \\
\text { (Major) }\end{array}$ & $\begin{array}{l}\{0,0,0,0\} \\
\text { (Minor) }\end{array}$ \\
\hline $\begin{array}{l}\text { Median value } \\
\qquad M_{\mathrm{i}, \mathrm{j}}[k]\end{array}$ & 1 & 0 & 0 & 1 \\
\hline
\end{tabular}

Table 1. An example of the median detection.

\subsection{Circuit Configuration}

Figure 2 illustrates the block diagram of the proposed CMOS median filter. As you can see from figure 2, the proposed median filter consists of bit-comparators (BCs) and majority circuits (MCs). In figure 2, the median detection is performed by a bit-comparison-based technique in order to realize small hardware cost.

Figure 3 illustrates the logic circuit (LC) in the bitcomparator. The logic circuit of figure 3 is designed to realize the following bit-comparison:

$$
L_{i}[k]=S 0_{i}[k] \cdot S 1_{i}[k]+P_{i}[k] \cdot \overline{S 1_{i}[k]},
$$

where

$$
\begin{aligned}
S 0_{i}[k+1]= & S 0_{i}[k] \cdot S 1_{i}[k] \\
& +\overline{M_{i}[k]} \cdot \overline{S 1_{i}[k]} \cdot P_{i}[k]
\end{aligned}
$$


and $\quad S 1_{i}[k+1]=M_{i}[k] \oplus P_{i}[k]+S 1_{i}[k]$.

In (3)-(5), $M_{i}[k]$ is the $k$-th bit of the median output and $L_{i}[k]$ is the $k$-th bit of the $\mathrm{BC}$ output.

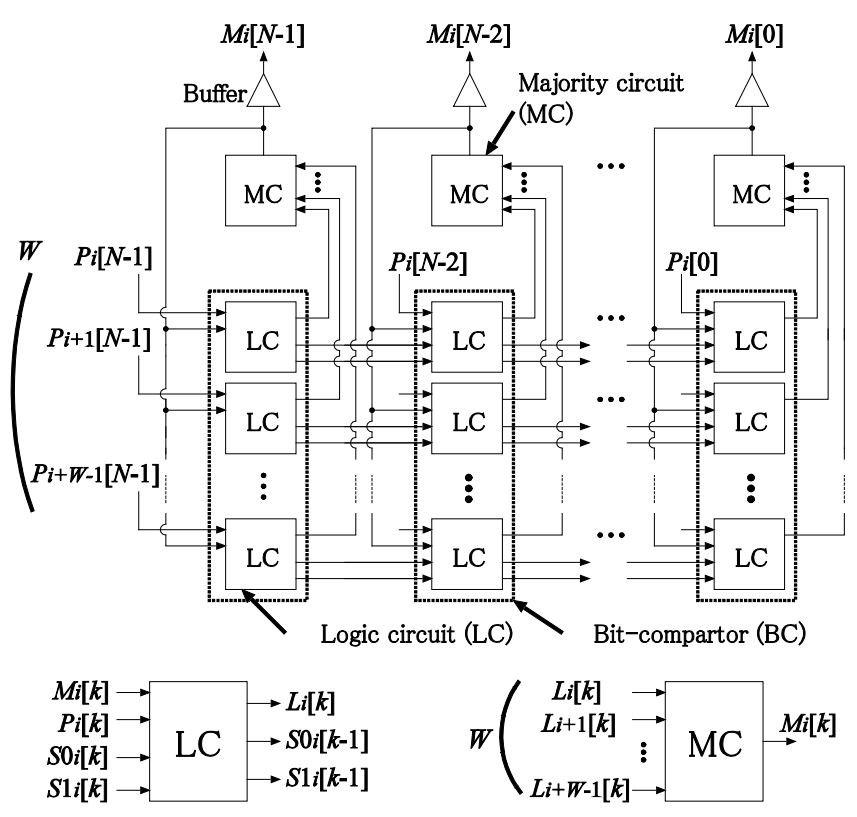

Fig. 2. Block diagram of CMOS median filter.

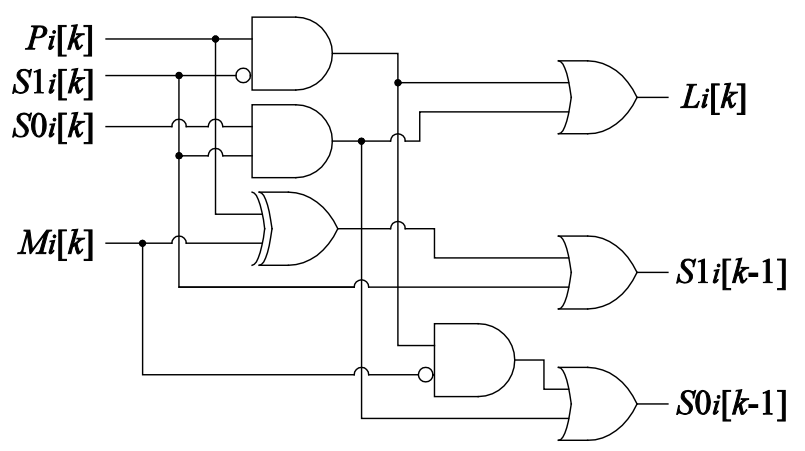

Fig. 3. Block diagram of a logic circuit.

The majority of $L_{i}[k]$ is detected in the majority circuit (MC). In the proposed median filter, the majority circuit is designed by using clocked neuron CMOS inverters. Figure 4 illustrates the proposed majority circuit using a clocked neuron CMOS inverter, where $C_{p}$ denotes the parasitic capacitance. When the clock $\Phi$ is in a high level, the clocked neuron CMOS inverter behaves as a traditional neuron CMOS inverter ${ }^{(8-10)}$. In this timing, the floating gate voltage $V_{F i}[k]$ of the clocked neuron inverter is given by

$$
V_{F i}[k]=\frac{\sum_{j \in W} L_{j}[k] C_{u}+V_{d d} C_{F N}}{W C_{u}+C_{F P}+C_{F N}},
$$

where $C_{u}$ is a unit capacitance between an input terminal and the floating gate, $C_{F N}$ is a capacitance between the floating gate and $N$-type region, and $C_{F P}$ is a capacitance between the floating gate and $P$-type region. In the proposed median filter, the unit capacitance $C_{u}$ is designed to satisfy the following conditions:

$$
C_{u} \gg C_{F N} \text { and } C_{F P}=C_{F N} .
$$

From (7), the floating gate voltage $V_{F i}$ can be rewritten as

$$
V_{F i}[k] \cong \sum_{j \in W} \frac{L_{j}[k]}{W},
$$

where

$$
\sum_{j \in W} L_{j}[k] \in\left\{0, V_{d d}, 2 V_{d d}, \cdots, W V_{d d}\right\} .
$$

From (8) and (9), we have

$$
M_{i}[k]=\left\{\begin{array}{ccc}
V_{d d}, & \text { if } & \sum_{j \in W} L_{j}[k] \leq \frac{W V_{d d}}{2} \\
0 . & \text { if } & \sum_{j \in W} L_{j}[k]>\frac{W V_{d d}}{2}
\end{array} .\right.
$$

As you can see from (10), the clocked neuron inverter behaves as a majority circuit when the clock $\Phi$ is in a high level. On the other hand, the clocked neuron inverter becomes high-impedance when the clock $\Phi$ is in a low level. In this timing, the output voltage is held in $C_{p}$. In other words, the proposed majority circuit behaves like a sample-and-hold circuit. Therefore, by adjusting the duty cycle of the clock pulse, the proposed median filter can reduce power consumption, because the through-current of the neuron inverter is suppressed.

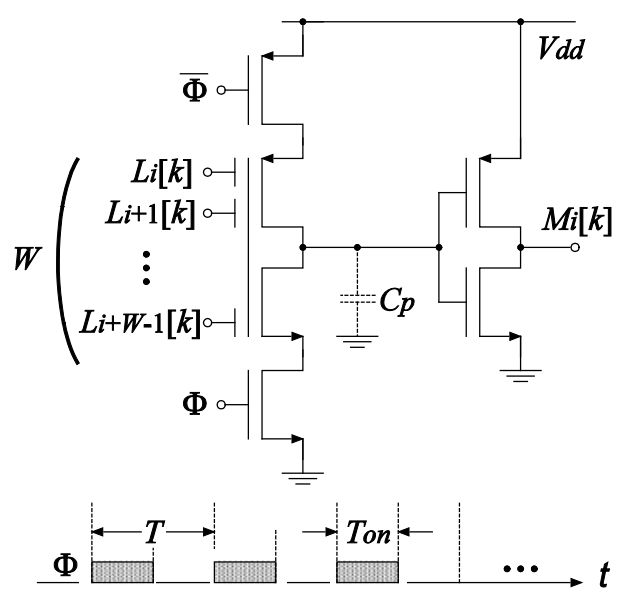

Fig. 4. Majority circuit using a locked neuron CMOS inverter. 


\section{Binarizing Circuit}

\subsection{Operation Principle}

After the median filtering, the input image is converted to a binary image and is resized to the image of $m^{\prime} \times n^{\prime}$ pixels. To obtain the pixel value of the resized image, $B_{i}(i=1, \ldots, m$, $\times n$ '), from the input image of $m \times n$ pixels, the following operation is performed:

$$
B_{i}=\left\{\begin{array}{ll}
1, \quad \text { if } & \frac{\sum_{j \in W^{\prime} l=1} \sum_{j}^{N} P_{j}[N-l] \cdot 2^{N-l}}{\sum_{j \in W^{\prime} l=1}^{N} \sum^{N-l}}>0.5 \\
0, & \text { if } \quad \frac{\sum_{j \in W^{\prime} l=1}^{N} P_{j}[N-l] \cdot 2^{N-l}}{\sum_{j \in W^{\prime} l=1}^{N} \sum^{N-l}} \leq 0.5
\end{array},\right.
$$

where $W^{\prime}$ is the window size to resize the input image. In other words, the resized image satisfies the following conditions:

$$
m^{\prime}=\frac{m}{W^{\prime}} \quad \text { and } \quad n^{\prime}=\frac{n}{W^{\prime}}
$$

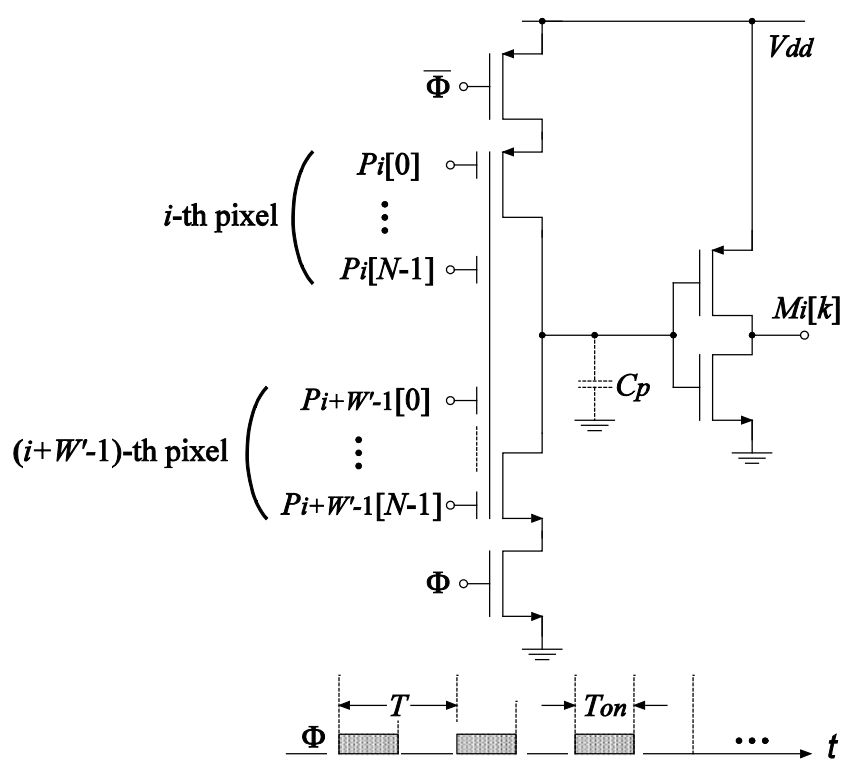

Fig. 5. Clocked neuron CMOS inverter.

\subsection{Circuit Configuration}

To achieve small power consumption and small hardware cost, clocked neuron MOSFET's are employed in the design of the proposed binarizing circuit. Figure 5 illustrates the proposed binarizing circuit using a clocked neuron CMOS inverter. In the proposed binarizing circuit, the floating gate capacitance is designed as $2^{N-1-k} \times C_{u}$ for $P_{i}[k]$. Owing to the binary-weighted floating capacitances, the proposed binarizing circuit requires no arithmetic logic circuit such as adder, multiplier, divider, and so on. Therefore, the proposed circuit can achieve simple circuit configuration.

In figure 5, the floating gate voltage $V_{F B i}$ of the clocked neuron inverter is given by

$$
V_{F B i}=\frac{\sum_{j \in W^{\prime} l=1} \sum_{u}^{N} C_{u} P_{j}[N-l] \cdot 2^{N-l}+V_{d d} C_{F N}}{\sum_{j \in W^{\prime}} \sum_{l=1}^{N} 2^{N-l} C_{u}+C_{F P}+C_{F N}},
$$

where the unit capacitance $C_{u}$ satisfies the condition of (7). From (7) and (13), we get

$$
V_{F B i}=\frac{\sum_{j \in W^{\prime} l=1} \sum_{j}^{N} P_{j}[N-l] \cdot 2^{N-l}}{\sum_{l=1}^{N} 2^{N-l} W^{\prime}},
$$

where

$$
P_{j}[k] \in\left\{0, V_{d d}\right\} .
$$

From (14) and (15), we have the pixel value of the resized image as follows:

$$
B_{i}=\left\{\begin{array}{cc}
\text { if } \quad & \frac{\sum_{j \in W^{\prime} l=1} \sum_{j}^{N} P_{j}[N-l] \cdot 2^{N-l}}{\sum_{l=1}^{N} 2^{N-l} W^{\prime}} \leq \frac{V_{d d}}{2} \\
V_{d d} . & \text { if } \quad \frac{\sum_{j \in W^{\prime} l=1}^{N} \sum_{j}^{N} P_{j}[N-l] \cdot 2^{N-l}}{\sum_{l=1}^{N} 2^{N-l} W^{\prime}}>\frac{V_{d d}}{2}
\end{array}\right.
$$

As you can see from (16), in spite of a simple circuitry, the proposed circuit can achieve not only binarization but also resizing. Furthermore, owing to the clocked neuron inverter, the proposed circuit can reduce power consumption by adjusting the duty cycle of the clock pulse.

\section{Simulation}

To clarify the characteristics of the proposed circuits, SPICE simulations are performed by assuming $0.35 \mu \mathrm{m}$ CMOS process. 


\subsection{CMOS Median Filter}

As an example of the proposed median filter, the 3bits/3-inputs proposed circuit was designed. Figure 6 demonstrates the simulated output of the proposed median filter, where the supply voltage is $1.8 \mathrm{~V}$ and the duty cycle is 0.5 . In figure 6 , the input voltages, $P_{1}, P_{2}$, and $P_{3}$, are as shown in Table 2. As you can see from figure 6 and Table 2, the proposed median filter can derive the median value $M_{i}$ from $P_{1}, P_{2}$, and $P_{3}$. Figure 7 shows the power consumption of the proposed median filter as a function of the duty cycle. In figure 7 , the blue-line shows the power consumption of the proposed median filter, the orange-line shows the power consumption of the conventional median filter using floating-gate-MOS-based low-power majority voting circuits ${ }^{(6)}$, and the grey line shows the conventional median filter using CMOS majority circuits ${ }^{(5)}$ shown in figure 8. As figure 7 shows, the proposed median filter can reduce the power consumption by controlling the duty cycle. Concretely, the proposed median filter reduced power consumption more than $37 \%$ from the conventional median filter ${ }^{(6)}$ when the duty cycle is 0.5 . Needless to say, the setting of duty cycle depends on the response speed of CMOS transistors.

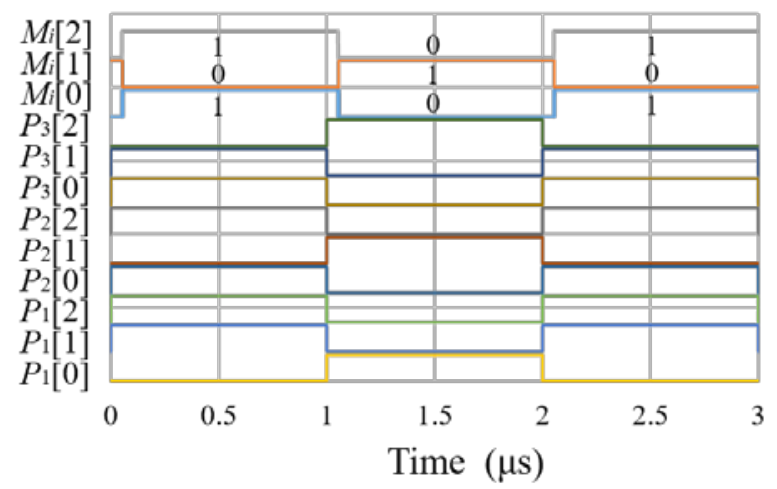

Fig. 6. Simulated output of the proposed median filter.

\subsection{Binarizing Circuit}

As an example of the proposed binarizing circuit using a clocked neuron CMOS inverter, the 4-bits/4-inputs proposed circuit was designed. Figure 9 demonstrates the simulated output of the proposed binarizing circuit, where the supply voltage is $1.8 \mathrm{~V}$ and the duty cycle is 0.5 . In figure 9, the input voltages, $P_{1}, P_{2}, P_{3}$ and $P_{4}$, are as shown in Table 3. As you can see from figure 9 and Table 3 , the proposed binarizing circuit can offer the pixel value of the resized image, $B_{i}$. Figure 10 shows the power consumption of the proposed circuit as a function of the duty cycle. In figure 10, the blue-line shows the power consumption of the proposed circuit with a clocked neuron CMOS inverter. On the other hand, the orange-line shows the power consumption of the proposed circuit without a clocked neuron CMOS inverter. As figure 10 shows, the proposed circuit with a clocked neuron CMOS inverter can reduce the power consumption by controlling the duty cycle. Concretely, more than $43 \%$ power consumption was reduced when the duty cycle is 0.5 .

Table 2. Setting of SPICE simulation.

\begin{tabular}{|c|c|c|c|}
\hline \multicolumn{3}{|c|}{ Time ( $\mu$ s) } \\
\hline From & 0 & 1.0 & 2.0 \\
\hline To & 1.0 & 2.0 & 3.0 \\
\hline Input & \multicolumn{3}{|c|}{ Value } \\
\hline$P_{1}$ & $\left\{V_{d d}, V_{d d}, 0\right\}$ & $\left\{0,0, V_{d d}\right\}$ & $\left\{V_{d d}, V_{d d}, 0\right\}$ \\
\hline$P_{2}$ & $(6)$ & $(1)$ & $(6)$ \\
\hline \multirow{2}{*}{$P_{3}$} & $\left\{0, V_{d d}, 0, V_{d d}\right\}$ & $\left\{0, V_{d d}, 0\right\}$ & $\left\{V_{d d}, 0, V_{d d}\right\}$ \\
\hline Output & $(3)$ & $(2)$ & $(5)$ \\
\hline$M_{i}$ & $\left\{V_{d d}, 0,0\right\}$ & $\left\{0, V_{d d}, V_{d d}\right\}$ \\
& $(5)$ & $(4)$ & $(3)$ \\
\hline
\end{tabular}

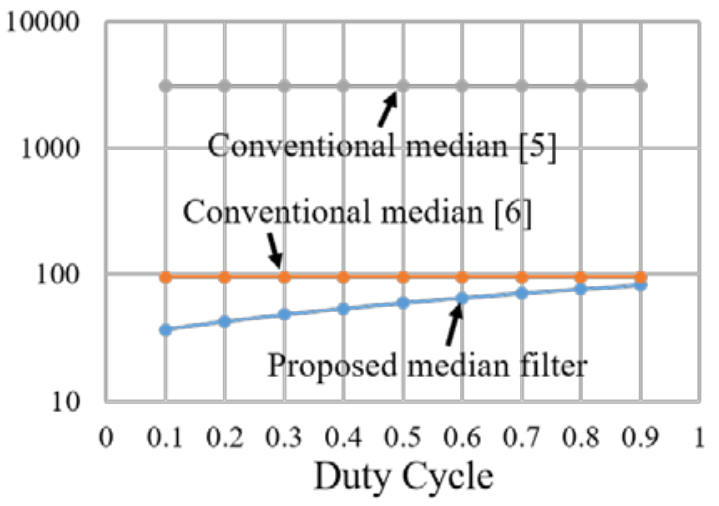

Fig. 7. Simulated power consumption of the median filter.

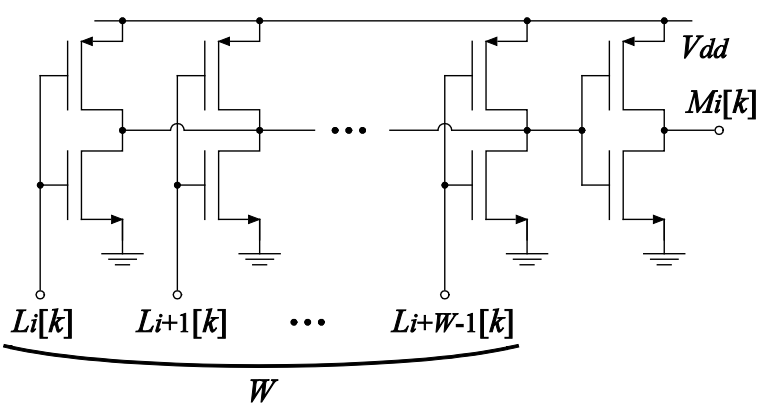

Fig. 8. Conventional CMOS majority circuit. 


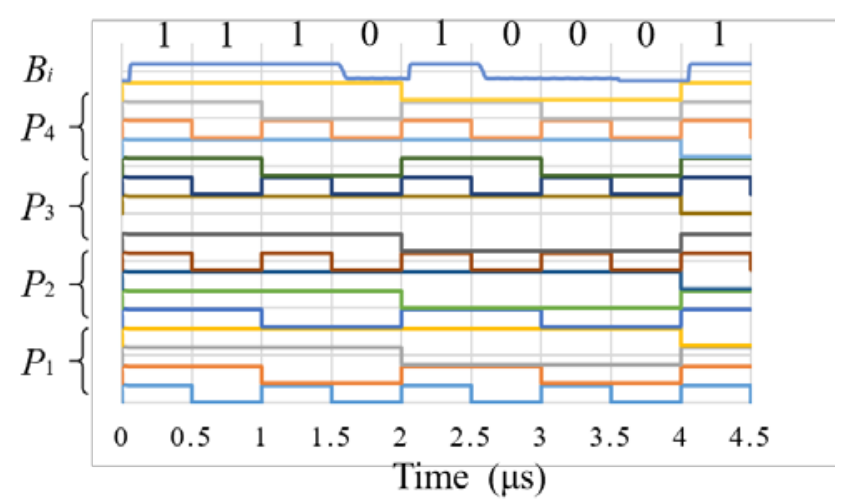

Fig. 9. Simulated output of the proposed binarizing circuit with a clocked neuron CMOS inverter.

Table 3. Setting of SPICE simulation.

\begin{tabular}{|c|c|c|c|c|c|c|c|c|c|}
\hline \multicolumn{10}{|c|}{ Time $(\mu \mathrm{s})$} \\
\hline From & 0 & 0.5 & 1.0 & 1.5 & 2.0 & 2.5 & 3.0 & 3.5 & 4.0 \\
\hline To & 0.5 & 1.0 & 1.5 & 2.0 & 2.5 & 3.0 & 3.5 & 4.0 & 4.5 \\
\hline Input & \multicolumn{10}{|c|}{ Value } \\
\hline$P_{1}$ & 15 & 7 & 11 & 3 & 13 & 5 & 9 & 1 & 14 \\
\hline$P_{2}$ & 15 & 14 & 7 & 6 & 11 & 10 & 3 & 2 & 13 \\
\hline$P_{3}$ & 15 & 13 & 14 & 12 & 7 & 5 & 6 & 4 & 11 \\
\hline$P_{4}$ & 15 & 11 & 13 & 9 & 14 & 10 & 12 & 8 & 7 \\
\hline Output & \multicolumn{10}{|c|}{ Value } \\
\hline$B_{i}$ & $V_{d d}$ & $V_{d d}$ & $V_{d d}$ & 0 & $V_{d d}$ & 0 & 0 & 0 & $V_{d d}$ \\
\hline
\end{tabular}

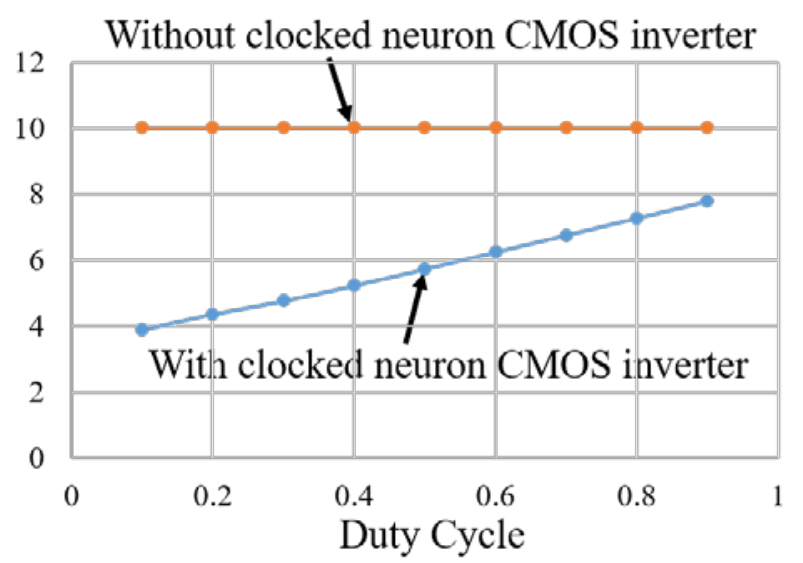

Fig. 10. Simulated power consumption of the binarizing circuit.

\section{Conclusions}

As a preprocessing circuit of the retinal prosthesis system, this paper has presented two types of image processing circuits: a median filter using clocked neuron
CMOS inverters and a binarizing circuit using a clocked neuron CMOS inverter. The SPICE simulations showed the following results:

1. By using clocked neuron CMOS inverters, the proposed median filter can offer median values without arithmetic logic circuits, such as adder, subtractor, multiplier, and divider.

2 . When the duty cycle is 0.5 , the proposed median filter can reduce about $37 \%$ power consumption from the conventional circuit.

3. By using a clocked neuron CMOS inverter with binary-weighted floating-capacitors, the proposed binarizing circuit can achieve simple circuit configuration. Concretely, the proposed circuit consists of 4 MOS transistors and a neuron CMOS inverter.

4. The proposed binarizing circuit achieve small power consumption. When the duty cycle was 0.5 , the proposed circuit reduced about $43 \%$ power consumption from the conventional circuit.

From these results, the proposed preprocessing circuits will be able to realize small power consumption. The following topics are left to a future study: 1 . Overall design of the retinal prosthesis system using the proposed preprocessing circuits, 2. Hardware implementation of the retinal prosthesis system, and 3. Performance evaluation of the experimental circuit such as circuit size, power consumption, response speed, etc.

\section{References}

(1) Takashi Tokuda, Kohei Hiyama, Shigeki Sawamura, Kiyotaka Sasagawa, Yasuo Terasawa, Kentaro Nishida, Yoshiyuki Kitaguchi, Takashi Fujikado, Yasuo Tano, and Jun Ohta : “CMOS-based multichip networked flexible retinal stimulator designed for image-based retinal prosthesis”, IEEE Trans. Electron Devices, Vol. 56, No. 11, pp.2577-2585, 2009

(2) Hideki Naganuma, Kouji Kiyoyama, and Tetsu Tanaka : “A $37 \times 37$ pixels artificial retina chip with edge enhancement function for 3-D stacked fully implantable retinal prosthesis”, Proc. of 2012 IEEE Biomedical Circuits and Systems Conference, pp.212-215, 2012

(3) Chih-Lin Lee and Chih-Cheng Hsieh : “A 0.5 V/1.8 V high dynamic range CMOS imager for artificial retina applications”, IEEE Sensors Journal, Vol. 15, No. 12, pp.6833-6838, 2015

(4) Ercan Kalali and Ilker Hamzaoglu : “A low energy 2D adaptive median filter hardware”, Proc. of 2015 Design, 
Automation \& Test in Europe Conference \& Exhibition, pp. 725-729, 2015

(5) Chang Long Lee and Chein-Wei Jen : “A bit-level scalable median filter using simple majority circuit”, Proc. of 1989 International Symposium on VLSI Technology, Systems and Applications, pp. 174-177, 1989

(6) Hideo Yamasaki and Tadashi Shibata : “A high-speed median filter VLSI using floating-gate-MOS-based lowpower majority voting circuits", Proc. of the 31st European Solid-State Circuits Conference, pp.125-128, 2005

(7) Masaaki Fukuhara and Masahiro Yoshida : "Power consumption of a Hamming distance search CAM using neuron MOS transistors", Proc. of 2006 IEEE International Symposium on Circuits and Systems, pp. 4345-4348, 2006

(8) Yujiro Harada, Kuniaki Fujimoto, Mitsutoshi Yahara, and Kei Eguchi : “A study on flash type A/D converter using neuron CMOS inverter”, Advanced Materials Research, Vol. 931-932, pp.915-919, 2014

(9) Yujiro Harada, Kei Eguchi, and Kuniaki Fujimoto : “A size reduction technique for an $\mathrm{A} / \mathrm{D}$ converter using neuron CMOS inverters”, Journal of Applied Science and Agriculture, Vol. 9, No. 11, pp.132-137, 2014

(10) Yujiro Harada, Kuniaki Fujimoto, Masaaki Fukuhara, and Masahiro Yoshida : "A minimum hamming distance search associative memory using neuron CMOS inverters", IEEJ Trans. Electronics, Information and Systems, Vol. 136, No. 1, pp. 36-42, 2016 